\title{
Downregulation of LINC00115 inhibits the proliferation and invasion of lung cancer cells in vitro and in vitro
}

\author{
Lili Shao ${ }^{1 \#}$, Qiongzhu Yu ${ }^{2 \#}$, Xiaomin Lu ${ }^{3 \#}$, Xiaodong Zhang ${ }^{1}$, Zhixiang Zhuang ${ }^{4}$ \\ ${ }^{1}$ Department of Medical Oncology, Tumor Hospital Affiliated to Nantong University, Nantong, China; ${ }^{2}$ Department of Pathology, The Affiliated \\ Changshu Hospital of Xuzhou Medical University, Suzhou, China; ${ }^{3}$ Department of Medical Oncology, The Affiliated Haian Hospital of Nantong \\ University, Nantong, China; ${ }^{4}$ Department of Medical Oncology, The Second Affiliated Hospital of Soochow University, Suzhou, China \\ Contributions: (I) Conception and design: L Shao; (II) Administrative support: None; (III) Provision of study materials or patients: L Shao, Q Yu; (IV) \\ Collection and assembly of data: Z Zhuang, X Zhang; (V) Data analysis and interpretation: X Lu; (VI) Manuscript writing: All authors; (VII) Final \\ approval of manuscript: All authors. \\ \#These authors contributed equally to this work. \\ Correspondence to: Zhixiang Zhuang. Department of Medical Oncology, The Second Affiliated Hospital of Soochow University, 1055 SanXiang \\ Road, Gusu District, Suzhou 215004, China. Email: 13951106391@139.com; Xiaodong Zhang. Department of Medical Oncology, Tumor Hospital \\ Affiliated to Nantong University, Nantong 226361, China. Email: $419317110 @$ qq.com.
}

\begin{abstract}
Background: Lung cancer is a common malignant tumor in clinical practice. Its morbidity and mortality rank first among malignant tumors. However, the pathogenesis of lung cancer has not been fully clarified. This study found that LINC00115 is highly expressed in lung cancer tissues, but the role and molecular mechanisms of LINC00115 in the occurrence and progression of lung cancer are still unclear.

Methods: Fluorescence quantitative PCR was used to detect the expression of LINC00115 in lung cancer tissues and para-carcinoma tissues. Cell counting kit-8 (CCK-8), clone formation, and Transwell assays were used to detect the effects of LINC00115 knockdown on the proliferation, clone formation, invasion, and migration of lung cancer cells. Western blot was used to detect the effects of LINC00115 knockdown on the expression of epithelial-mesenchymal transition (EMT)-related molecules. Finally, a xenograft model in nude mice was used to detect the effect of LINC00115 knockdown on the proliferation of lung cancer cells in vivo.

Results: Compared with para-carcinoma tissue, LINC00115 was highly expressed in lung cancer tissue. Cell function experiments showed that knockdown of LINC00115 could significantly inhibit the proliferation, invasion, and migration of lung cancer cells. Western blot results showed that knockdown of LINC00115 could significantly inhibit the expression of the EMT-related proteins N-cadherin, vimentin, and fibronectin, and promoted the expression of E-cadherin. In vivo experiments in nude mice showed that knockdown of LINC00115 could significantly inhibit the proliferation of lung cancer tissues in vivo.

Conclusions: LINC00115 is highly expressed in lung cancer tissues, and knockdown of LINC00115 can significantly inhibit the proliferation and invasion of lung cancer, which provides a theoretical basis for the design of targeted molecules for the subsequent treatment of lung cancer.
\end{abstract}

Keywords: LINC00115; lung cancer cells; proliferation; invasion

Submitted Jun 25, 2021. Accepted for publication Aug 05, 2021.

doi: 10.21037/atm-21-3724

View this article at: https://dx.doi.org/10.21037/atm-21-3724

\section{Introduction}

Lung cancer is one of the most common malignant tumors worldwide. According to the 2018 global cancer data released by the International Cancer Institute, the morbidity (11.6\%) and mortality (18.4\%) of lung cancer rank the highest of all malignant tumors $(1,2)$. In recent 
years, with the development of medical treatment and diagnosis technology, overall cancer mortality has declined, but the effect of lung cancer treatment is still not ideal. The main reasons are the low early diagnosis rate of lung cancer, limited treatment methods, and drug resistance, which lead to poor treatment efficacy and poor prognosis of lung cancer patients $(3,4)$. With the progress of clinical medicine and molecular biology technology, lung cancer has made great progress in diagnosis and treatment, especially the development of targeted drugs, which has significantly improved the treatment outcomes of lung cancer. However, there are still many uncertainties regarding the occurrence and progression mechanisms of lung cancer (5-7). Therefore, exploring the molecular mechanisms of lung cancer and finding new molecular markers that can be used as early diagnosis and treatment targets can provide new strategies for the diagnosis and treatment of lung cancer. This is of great significance to improve the survival time and quality of life of lung cancer patients, and also provides new ideas for clarifying the mechanisms of lung cancer.

Long non-coding RNA (lncRNA) is a kind of RNA that does not encode proteins. LncRNAs exceed $200 \mathrm{nt}$ in length and can regulate gene expression at various levels, including transcriptional and post-transcriptional regulation levels $(8,9)$. At present, a large number of studies have shown that IncRNAs play important regulatory roles in the occurrence and progression of various diseases, such as tumors, cardiovascular disease, obesity, and immune disorders (10-12). Previous studies have shown that lncRNAs are involved in the regulation of tumorigenesis and its development, and can be used as markers for early diagnosis and as targets for treatment $(13,14)$. For example, IncRNA H19 promotes lung cancer proliferation and metastasis by inhibiting the function of miR-200a (15). LncRNA SNHG11 is highly expressed in lung cancer tissues, and further promotes the proliferation and migration of cancer cells by activating the Wnt/ $\beta$-catenin signaling pathway (16). In addition, lncRNA RFPL $3 S$ is more highly expressed in lung cancer tissues than in normal tissues, and is significantly correlated with TNM stage and the Ki-67 labeling index. In the survival analysis, it was found that the upregulation of lncRNA RFPL3S expression is closely related to the poor survival of patients, and is negatively correlated with the progression of the disease, which shows that RFPL3S may have important clinical significance and can be used as a prognostic biomarker of lung cancer (17). LINC00115 is a newly identified non-coding RNA with important regulatory functions. Previous studies have shown that LINC00115 is highly expressed in triple-negative breast cancer (TNBC) tissues, and knocking down LINC00115 inhibits the migration and invasion of breast cancer cells. Molecular mechanism studies show that LINC00115 promotes breast cancer metastasis by regulating $m i R-7$ and KLF4 expression. In colorectal cancer, LINC00115 inhibits the $\mathrm{PI} 3 \mathrm{~K} / \mathrm{AKT} / \mathrm{mTOR}$ signaling pathway by downregulating miR-489-3p, highlighting the LINC00115/miR-489-3p axis as a potential therapeutic target for colorectal cancer metastasis (18). So antiLINC00115 compounds or agents consequently targeting the PI3K/AKT/mTOR pathway might serve as novel therapeutic strategies for the colorectal cancer treatment.

Numerous studies have showed that lncRNAs have been found to be involved in the underlying molecular mechanisms of lung cancer (14-16). Therefore, it is necessary and urgent to discover and identify novel lncRNAs and understand the function of lncRNAs. So far, the expression and regulatory function of LINC00115 in lung cancer tissue is not yet clear. Therefore, this study preliminarily analyzes the expression of LINC00115 in lung cancer tissue, and explores the effect of LINC00115 on the proliferation and migration of lung cancer cells and related molecular mechanisms. We present the following article in accordance with the ARRIVE reporting checklist (available at https://dx.doi.org/10.21037/atm-21-3724).

\section{Methods}

\section{Collection of clinical samples}

In this study, 52 patients with lung adenocarcinoma treated in our hospital from December 2018 to May 2020 were included, including 21 females and 31 males. Their ages ranged from 35 to 69 years old, with an average of $55.2 \pm 6.4$ years old. All patients underwent lung cancer resection, and tumor tissue samples and paired normal tissues (more than $5 \mathrm{~cm}$ away from the edge of cancer tissue) were obtained. All patients did not undergo any chemotherapy and radiotherapy before the operation, and the diagnosis was confirmed by three pathologists. All the samples were fresh and stored in liquid nitrogen immediately after being isolated.

All procedures performed in this study involving human participants were in accordance with the Declaration of Helsinki (as revised in 2013). The study was approved by Tumor Hospital Affiliated to Nantong University (No. 
20516215) and informed consent was taken from all the patients.

\section{Cell culture}

Lung cancer cell lines (A549, PC-9, H292, HCC-78, SPC-A-1) and the normal bronchial epithelial cell line 16HBE (Shanghai Institute of Biological Sciences, Shanghai, China) were cultured with RPMI-1640 or Dulbecco's modified Eagle medium (DMEM; Gibco, USA) containing $10 \%$ fetal bovine serum (FBS) in an incubator of $37{ }^{\circ} \mathrm{C}, 5 \%$ $\mathrm{CO}_{2}$, and $95 \%$ humidity. When the cell confluence reached about $70-80 \%$, the medium in the culture bottle was discarded. The cells were washed with phosphate-buffered saline (PBS) 2-3 times, trypsin was added for digestion, then cells were centrifuged at 1,000 rpm for 5 minutes. After removing the supernatant, the cells were resuspended in RPMI-1640 medium containing 10\% FBS and $100 \mu \mathrm{M}$ penicillin and streptomycin. The cells were counted for subsequent culture and treatment.

\section{Reverse transcription-PCR (RT-PCR)}

Total RNA was extracted from cells and tissues by the Trizol kit (Shanghai Enzyme Link Biotechnology Co., Ltd.). The purity and concentration of RNA were measured by the Nanodrop 2000 spectrophotometer. RNA was reverse transcribed to cDNA using the reverse transcriptase kit and MMLV Reverse. It was then amplified by an amplification kit and PCR, and was tested using the ABI StepOne Plus system. The relative expression levels of each gene were calculated by the $2^{-\Delta \Delta \mathrm{Ct}}$ method, where $\Delta \mathrm{Ct}=\mathrm{Ct}$ value of target gene - Ct value of internal reference gene, $\Delta \Delta \mathrm{Ct}=$ transfection group $\Delta \mathrm{Ct}-$ control group $\Delta \mathrm{Ct}$. The cycling conditions were: $95^{\circ} \mathrm{C}$ for 10 minutes, then $95{ }^{\circ} \mathrm{C}$ for $10 \mathrm{~s}$ and $60{ }^{\circ} \mathrm{C}$ for $40 \mathrm{~s}, 40$ cycles. Primer sequences of LINC00115 and the internal reference gene GAPDH were generated from the literature (19).

LINC00115:

Forward: 5'-TGGCTTGTCTTCCATCGTCC-3';

Reverse: 5'-GCACGAGGGTTGTTACAGGA-3'.

GAPDH:

Forward: 5'-ACCACAGTCCATGCCATCAC-3';

Reverse: 5'-TCCAC CCTGTTGCTGTA-3'.

\section{Transfection experiment}

Control shRNA (5'-TTCTCCGAACGTGTCACGT-3'),
shRNA-1 (5'-GAAGAAUGGUACAAAUCCAAG-3'), and shRNA-2 (5'-CUUAAAGGAACCAAUGAGUCC-3') used in transfection were synthesized, packaged, and their titer was determined by Sangon, Shanghai. Twenty-four hours before transfection, PC-9 cells in logarithmic growth phase were inoculated into a 6 -well plate $\left(4 \times 10^{5} /\right.$ well $)$ and gently shaken to evenly distribute the cells. Two hours before transfection, the original culture medium was removed and replaced with fresh basic culture medium without antibiotics and serum. After dilution, the lentivirus was added into the corresponding culture well, shaken gently and mixed well, and then cultured in the incubator. After 12 hours, the transfection mixture was removed and replaced with fresh culture medium, then the culture plate was cultured in the incubator for 48 hours.

\section{Cell counting kit-8 (CCK-8) assay for cell viability}

First, 48 hours after transfection, cells were seeded into 96well plates $(1,000$ cells/well) and cultured in an incubator of $37{ }^{\circ} \mathrm{C}, 5 \% \mathrm{CO}_{2}$, and $95 \%$ humidity. After incubation for 24, 48, 72, and 96 hours, diluted CCK-8 solution was added for the reaction. Then, the absorbance value [optical density (OD) value] was detected at $450 \mathrm{~nm}$ by a microplate analyzer, and the cell growth curve was generated.

\section{Scratch test}

First, 48 hours after transfection, cells were seeded into 6-well plates. After 12 hours, the confluence rate was about $80-90 \%$. The tip of the pipette $(200 \mu \mathrm{L})$ was used to scratch along the straight edge while the tip was vertical. The plates were washed 3 times with PBS to remove the cells. The culture medium was put in an incubator of $37^{\circ} \mathrm{C}, 5 \% \mathrm{CO}_{2}$, and $95 \%$ humidity, and images and measurements were taken under an inverted microscope at 0 and 24 hours.

\section{Invasion and migration assay}

The presence or absence of Matrigel coating was used to evaluate cell migration and invasion in a Transwell chamber $(0.8 \mu \mathrm{m})$. First, the treated cells $\left(3 \times 10^{5} /\right.$ well $)$ were placed in the upper chamber and $500 \mu \mathrm{L}$ DMEM medium (20\% FBS) was added in the lower chamber. After incubation for 24 hours at $37{ }^{\circ} \mathrm{C}, 5 \% \mathrm{CO}_{2}$, and $95 \%$ humidity, the cells were fixed with $1 \%$ paraformaldehyde for 10 minutes and washed 3 times with PBS. After the non-invasive and non-migratory cells were removed with 
cotton swabs, they were stained with $0.1 \%$ crystal violet. Finally, the invading and migrating cells were calculated under the inverted microscope.

\section{Western blot}

The transfected cells were collected, and RIPA lysis buffer (Beyotime, Shanghai, China) was added to extract the total protein. The total protein concentration was detected using the BCA protein detection kit (Beyotime). The separation gel and concentrated gel were prepared by conventional methods. The sample was added to the gel hole with $70 \mu \mathrm{g}$ per lane. Electrophoresis was performed at a constant setting of $80 \mathrm{~V}$ (concentrated gel)/120 V (separation gel) until bromophenol blue reached the gel edge. The total protein was isolated by SDS-PAGE and transferred to PVDF membrane. The sample was blocked with $5 \%$ skim milk powder at room temperature for 2 hours, then incubated overnight with primary antibodies as following: anti-Ncadherin (Abcam, 1:1,000), anti-E-cadherin (CST, 1:800), anti-vimentin (Abcam, 1:1,000), anti-fibronectin (CST, 1:1,000) and anti-beta-actin (CST, 1:1,500). The membrane was then washed 3 times with PBS for 5 minutes each time. The membrane was incubated with the second antibody at room temperature for 2 hours, then washed with trisbuffered saline with $0.1 \%$ Tween 20 (TBST). Enhanced chemiluminescence (ECL) developer A and B were mixed in equal volume and added to the polyvinylidene difluoride (PVDF) membrane for exposure and imaging.

\section{In vivo experiment in nude mice}

Eight male BALB/C nude mice (4-6 weeks old) (SIPPRBK, Shanghai, China) were kept in miniature isolation cages. The transfected PC-9 cells were collected and digested with trypsin, and the number of living cells was calculated and adjusted to $10^{7} / \mathrm{mL}$. A $100 \mu \mathrm{L}$ cell suspension was taken and injected subcutaneously into the lateral abdomen of the mice. The tumor size was observed on the 5th day after injection, and the tumor volume was measured with a vernier caliper. The tumor volume was recorded every 5 days. The nude mice were sacrificed on the 35 th day, and the tumor-bearing tissues were collected for imaging and weighing.

Experiments were performed under a project license (No.: 51815674) granted by Tumor Hospital Affiliated to Nantong University, in compliance with Chinese guidelines for the care and use of animals.

\section{Statistical analysis}

SPSS 19.0 was used to analyze the data. Measurement data were expressed as mean \pm standard deviation. Measurement data with a normal distribution were compared by the $t$-test or analysis of variance (ANOVA), and measurement data with a non-normal distribution were compared by the nonparametric rank-sum test. Count data were compared by the chi-square test. $\mathrm{P}<0.05$ was statistically significant.

\section{Results}

\section{LINC00115 was highly expressed in lung cancer tissues and correlated with the clinicopathological features of patients}

To explore the expression of LINC00115 in lung cancer tissues, the published non-coding RNA sequencing data of lung cancer from The Cancer Genome Atlas (TCGA) database were analyzed. The results showed that the expression level of LINC00115 in lung cancer tissues was significantly higher than that in normal tissues $(\mathrm{P}<0.01)$ (Figure 1A). Further correlation analysis of clinicopathological features showed that LINC00115 expression was closely related to the T stage (T1-T2/T3-T4) and TNM stage (stage III-V/stage I-II) of lung cancer patients (Figure 1B,1C). At the same time, the survival analysis based on the above data showed that lung cancer patients with high expression of LINC00115 had a shorter survival time, with a significant difference $(\mathrm{P}<0.05)$ (Figure 1D). These results suggest that the expression level of LINC00115 can be used as a marker for the diagnosis and prognosis of lung cancer.

To further analyze the expression of LINC00115, the expression of LINC00115 in 52 cases of lung cancer tissues and paracancerous tissues was detected by fluorescence quantitative PCR. The results showed that the expression of LINC00115 in lung cancer tissues was significantly higher than that in paracancerous tissues $(\mathrm{P}<0.01)$ (Figure 2A). Further analysis of lung cancer cell lines showed that the expression level of LINC00115 in lung cancer cells was significantly higher than that in normal bronchial epithelial cells (16-HBE), with the highest increase in PC-9 cells (Figure 2B). To further explore the specific function of LINC00115 in lung cancer cells, the lentivirus knockdown vector was constructed according to the gene sequence of LINC00115, and then transfected into PC-9 cells. The experiment included four groups: blank control group (control), lentiviral interference blank control group (control shRNA), LINC00115 interference sequence 1 (shRNA-1), and LINC00115 interference sequence 2 (shRNA-2). The 
A

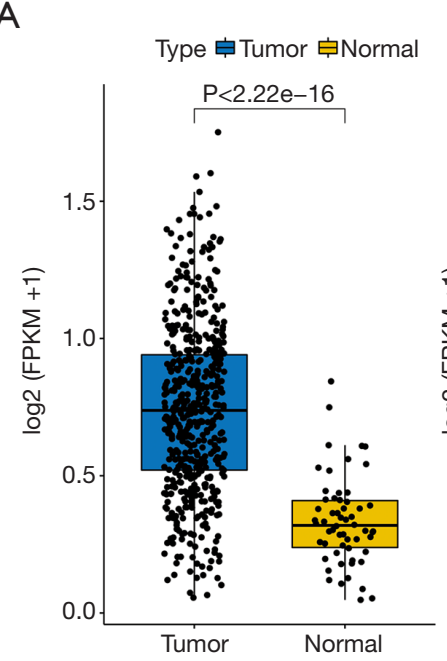

B

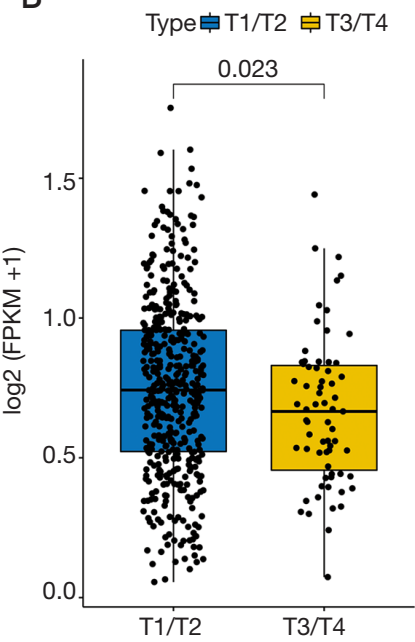

C

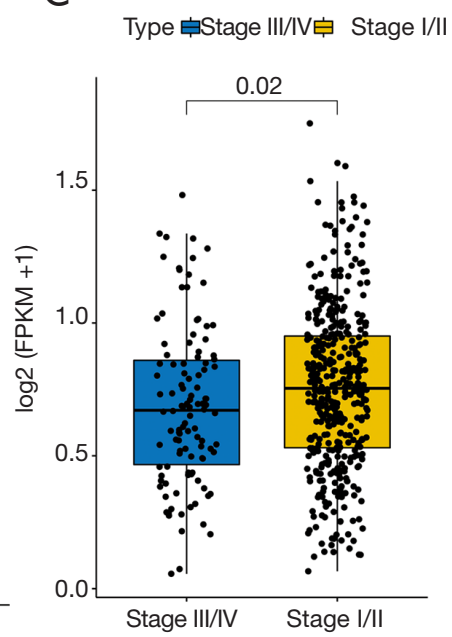

D

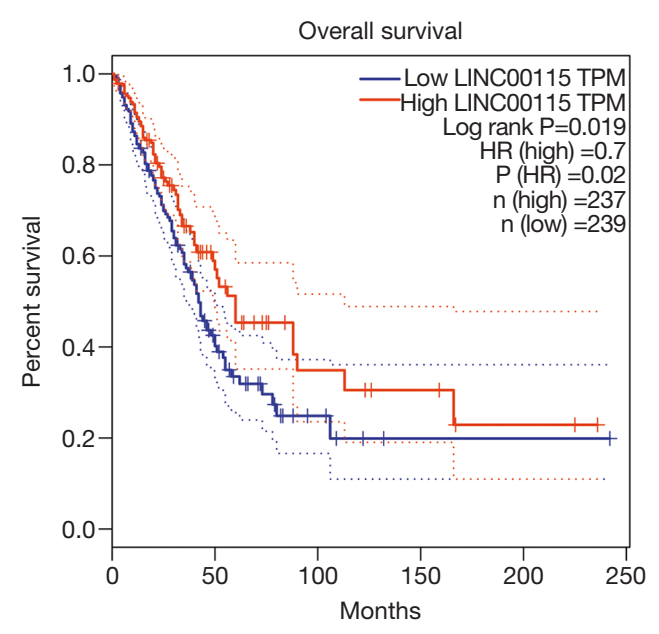

Figure 1 LINC00115 is highly expressed in lung cancer tissues and is correlated with clinicopathological features. (A) Expression of LINC00115 in lung cancer tissues and para-carcinoma tissues in TCGA database. (B,C) Correlation analysis between LINC00115 expression and the clinicopathological features of patients. (D) Relationship between LINC00115 expression in lung cancer tissues and the survival time of patients. TCGA, The Cancer Genome Atlas; TPM, transcripts per million.

results showed that compared with the control group, the control shRNA group was not significantly different in terms of the expression of LINC00115 in cells. Compared with the control shRNA group, transfection of lentivirus containing LINC00115 interference shRNA-1 and shRNA-2 significantly knocked down the expression level of LINC00115 in PC-9 cells (Figure 2C).

\section{Knockdown of LINC00115 can significantly inbibit the proliferation and clone formation of lung cancer PC-9 cells}

To further study the specific biological function of
LINC00115 in lung cancer cells, the effect of LINC00115 knockdown on the proliferation of lung cancer PC-9 cells was detected by the CCK- 8 assay. The results showed that there was no significant difference between the control group and the control shRNA group in terms of the proliferation of lung cancer PC-9 cells. Compared with the control shRNA group, the shRNA-1 and shRNA-2 groups had significantly inhibited proliferation of PC-9 cells 48 hours after transfection. With the extension of culture time (72 hours), the inhibition effect of shRNA-2 was more significant than that of shRNA-1 (Figure 3A). Compared with the control shRNA group, the colony formation ability 

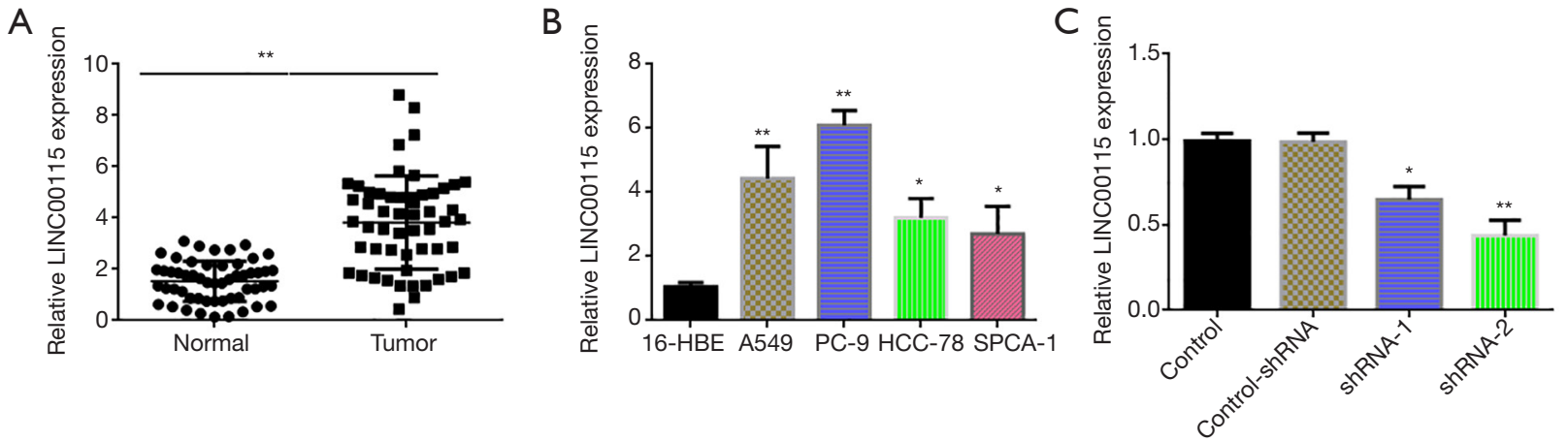

Figure 2 LINC00115 is highly expressed in lung cancer tissues and cells. (A) Expression of LINC00115 in 52 cases of lung cancer tissues and para-carcinoma tissues. (B) Fluorescent quantitative PCR detection of the expression of LINC00115 in lung cancer cell lines. (C) Fluorescence quantitative PCR detection of the efficiency of LINC00115 knockdown in lung cancer PC-9 cells. *, $\mathrm{P}<0.05 ;{ }^{* *}, \mathrm{P}<0.01$.

A

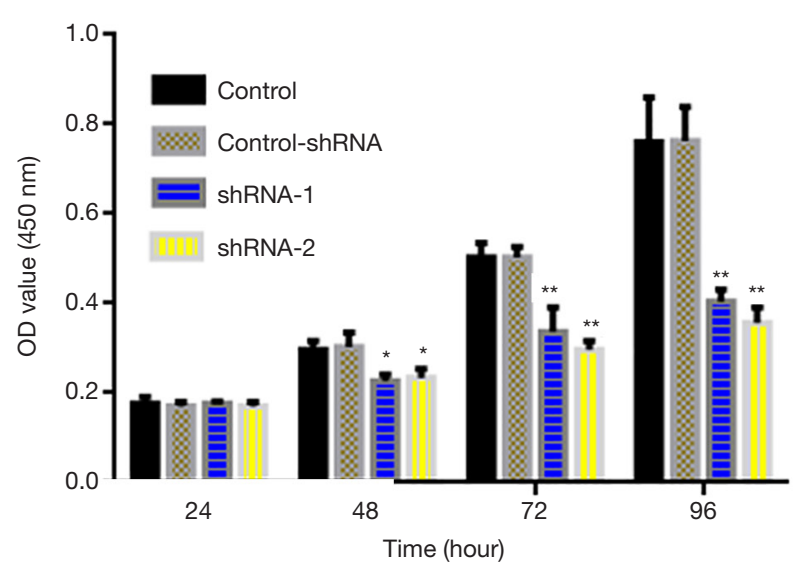

B
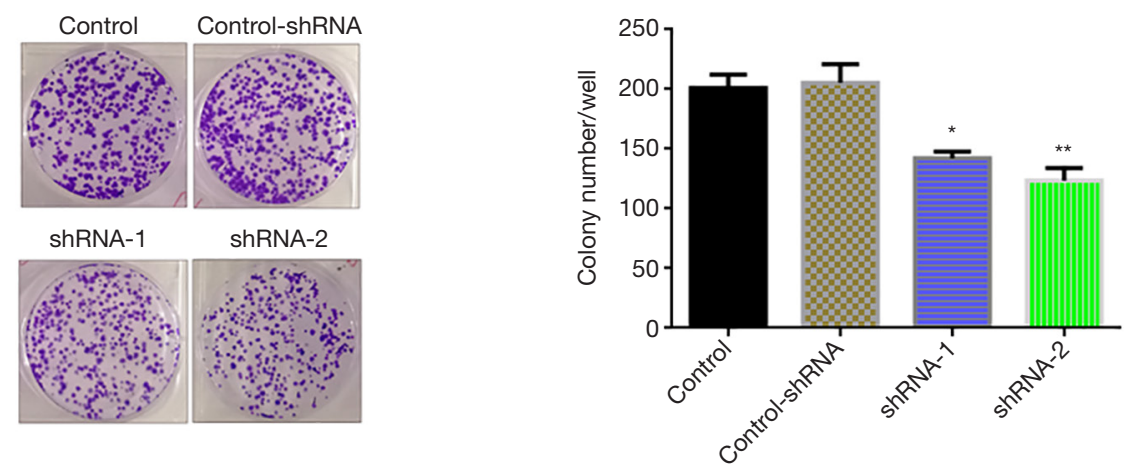

Figure 3 Knockdown of LINC00115 can significantly inhibit the proliferation of lung cancer cells. (A) The effect of LINC00115 knockdown on the proliferation of lung cancer cells detected by the CCK-8 assay. (B) The effect of LINC00115 knockdown on the clone formation of lung cancer cells. They were stained with $0.1 \%$ crystal violet. *, $\mathrm{P}<0.05 ;{ }^{* *}, \mathrm{P}<0.01$. CCK-8, cell counting kit-8; OD, optical density. 
A
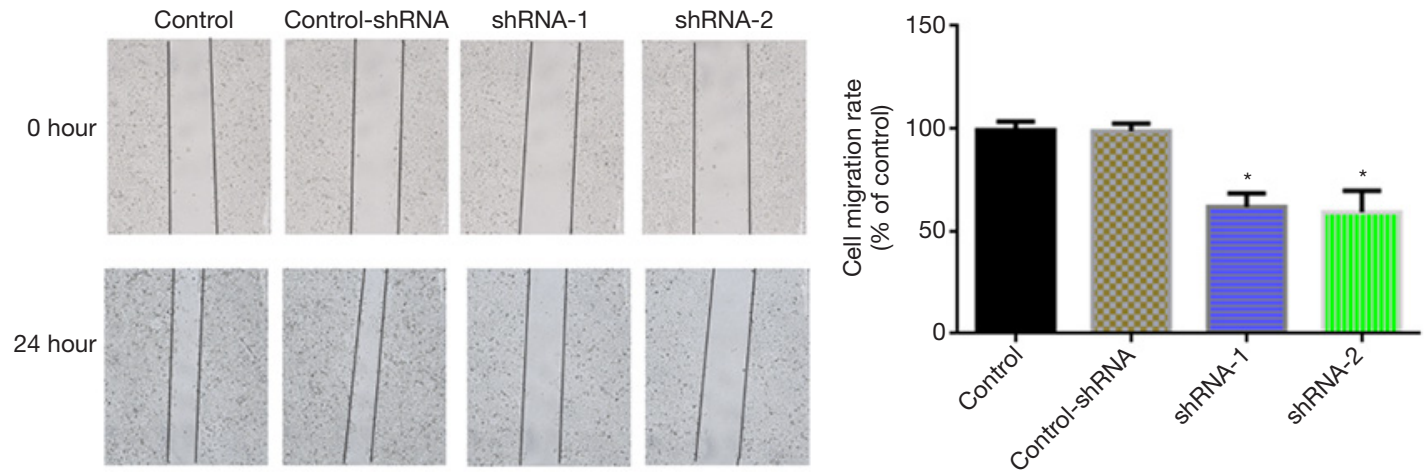

B
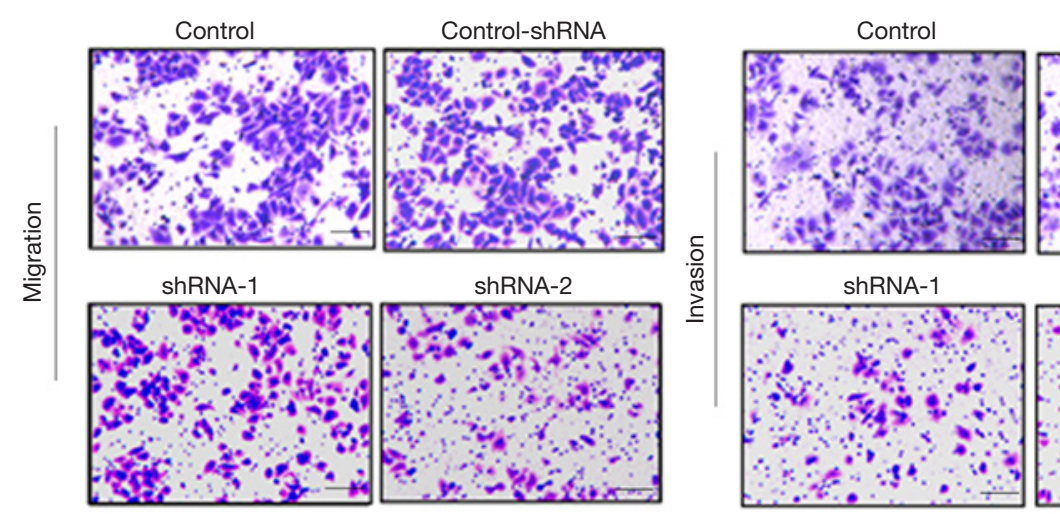

Control-shRNA
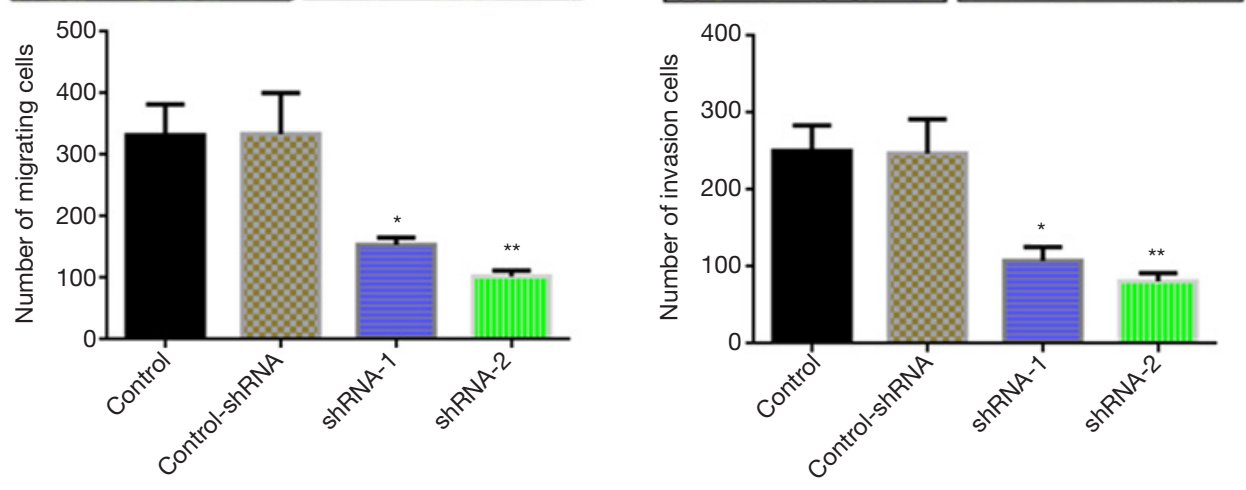

Figure 4 Knockdown of LINC00115 inhibits the migration and invasion of lung cancer cells. (A) Scratch test to detect the migration of lung cancer cells. (B) Transwell assay to detect the invasion and migration of lung cancer cells (100×). They were stained with $0.1 \%$ crystal violet. Scale bar represent $50 \mu \mathrm{m} .{ }^{*}, \mathrm{P}<0.05 ;{ }^{* *}, \mathrm{P}<0.01$.

of PC-9 cells was significantly inhibited in the shRNA-1 and shRNA-2 groups after 15 days of culture (Figure $3 B$ ). These results indicated that knockdown of LINC00115 can significantly inhibit the proliferation of lung cancer cells.

\section{Knockdown of LINC00115 can inhibit the invasion and migration of lung cancer cells}

The effects of LINC00115 on the invasion and migration of lung cancer cells were further studied by the scratch test and Transwell assay. Scratch test results showed that the migration ability of PC-9 cells was significantly decreased after knockdown of LINC00115 (shRNA-1 and shRNA-2) compared with the control shRNA group (Figure 4A). The Transwell assay showed that compared with the control shRNA group, the migration and invasion ability of the shRNA-1 and shRNA-2 groups were significantly decreased (Figure 4B). These results indicate that knockdown of 

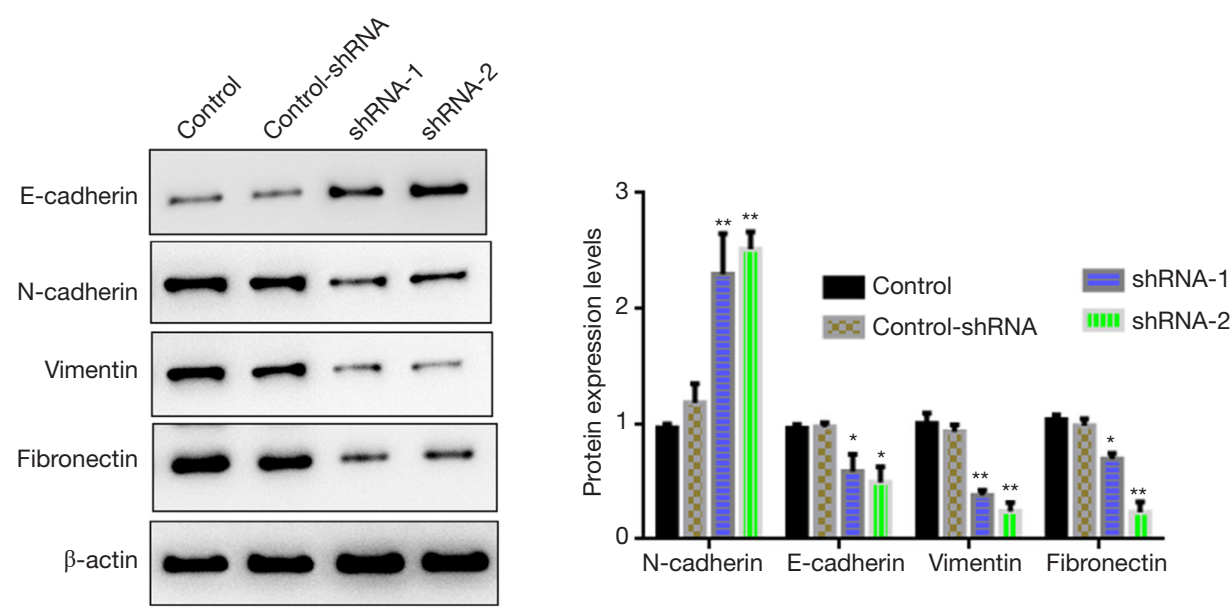

Figure 5 Effect of LINC00115 knockdown on invasion and migration-related protein expression of lung cancer PC-9 cells. *, P<0.05; **, $\mathrm{P}<0.01$.

LINC00115 can significantly inhibit the invasion and migration of lung cancer cells.

\section{Elimination of LINC00115 can inbibit the expression of molecular proteins associated with epithelial-mesenchymal transition (EMT) in lung cancer cells}

To further study the molecular mechanisms of LINC00115 in lung cancer cells, western blot was performed. The results showed that there was no significant difference between the control group and the control shRNA group in terms of the expression of invasion and migration-related proteins of lung cancer PC-9 cells. However, knockdown of LINC00115 could significantly promote the expression of $\mathrm{E}$-cadherin and inhibit the expression of $\mathrm{N}$-cadherin, vimentin, and fibronectin (Figure 5).

\section{Knockdown of LINC00115 can inbibit the proliferation of lung cancer cells in vivo}

To further determine the effect of LINC00115 knockdown on the proliferation of lung cancer cells in vivo, the transfected PC-9 cells were injected subcutaneously into nude mice. The nude mice were sacrificed on the 35 th day and the tumor tissues were collected (Figure $6 A$ ). The growth curve was generated according to the measured volume of tumor-bearing tissue. The results showed that the growth rate of transplanted tumors in the shRNA-2 group was significantly lower than that in the control group and control shRNA group (Figure $6 B$ ) (both $\mathrm{P}<0.01$ ). The weight of the transplanted tumor in each nude mouse was weighed and the average value was calculated. The results showed that the weight of transplanted tumors in the shRNA-2 group was significantly lower than that in the control group and control shRNA group (Figure 6C). In addition, the expression level of LINC00115 in the shRNA-2 group was significantly lower than that in the control group and control shRNA group (Figure 6D). These results indicate that knockdown of LINC00115 expression can significantly inhibit the growth rate of transplanted tumors in nude mice.

\section{Discussion}

At present, a large number of studies have shown that lncRNAs play important roles in the occurrence and metastasis of lung cancer, and these lncRNAs participate in physiological and biochemical functions through regulating gene expression and post-transcriptional protein translation (20-22). Therefore, identification of novel lncRNAs and elucidation of their physiological regulatory functions have important theoretical and clinical significance for the early diagnosis and targeted therapy of lung cancer. In this study, we first found that LINC00115 was highly expressed in lung cancer tissues, which was correlated with the clinicopathological grade of patients. In vitro cell experiments showed that knockdown of LINC00115 could significantly inhibit the proliferation, clone formation, invasion, and migration of lung cancer cells. In addition, knockdown of LINC00115 significantly 
A

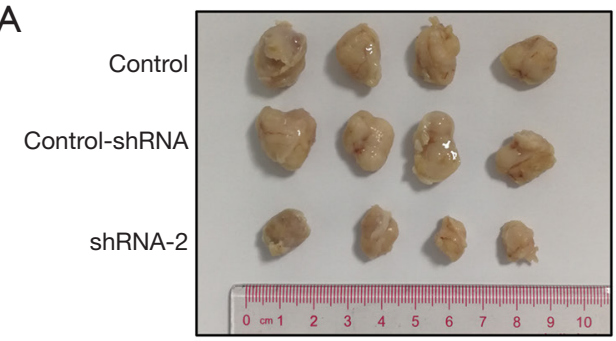

C

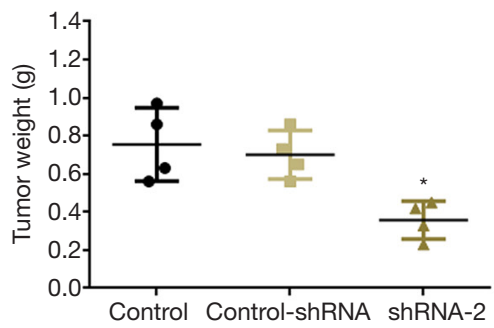

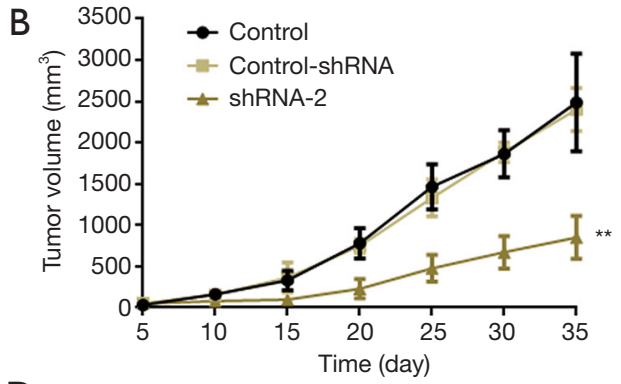

D

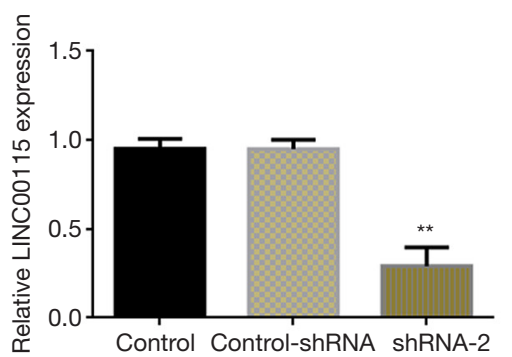

Figure 6 Effect of LINC00115 knockdown on the proliferation of lung cancer PC-9 cells in vivo. (A) Shape and size of the tumor-bearing tissue after subcutaneous inoculation for 5 days. (B) Volume of the tumor-bearing tissue was monitored and calculated after subcutaneous inoculation for 5 days. (C) Collected tumor-bearing tissue was weighed. (D) Expression of LINC00115 in the tissue was detected by fluorescence quantitative PCR. *, $\mathrm{P}<0.05 ;{ }^{* *}, \mathrm{P}<0.01$.

promoted the expression of E-cadherin and inhibited the expression of $\mathrm{N}$-cadherin, vimentin, and fibronectin. In vivo experiments further confirmed that knockdown of LINC00115 could significantly inhibit the proliferation of lung cancer cells in vivo.

One of the main characteristics of tumor cells is their unlimited malignant proliferation. Previous studies have shown that lncRNAs can regulate the proliferation of tumor cells and are associated with the poor prognosis of lung cancer. For example, the expression of lncRNA AC020978 is upregulated in lung cancer and is associated with advanced TNM stage and poor prognosis, and functional analysis shows that lncRNA AC020978 can promote cell proliferation (23). In another example, lncRNA EPEL is associated with the recurrence and survival of lung cancer patients and promotes lung cancer cell proliferation through the activation of E2F target genes (24). This study found that LINC00115 was highly expressed in lung cancer cell lines, and TCGA data analysis showed that the expression level of LINC00115 was closely related to the T stage and TNM stage of lung cancer patients, and affected the survival time of lung cancer patients. Therefore, the expression level of LINC00115 can be used as a marker for the diagnosis and prognosis of lung cancer. In addition, cell function experiments showed that knockdown of LINC00115 could significantly inhibit the proliferation and clone formation ability of lung cancer cells, and in vivo experiments further proved that knockdown of LINC00115 could significantly inhibit the proliferation ability of lung cancer cells.

EMT is an important process of tumor cell metastasis. A large number of studies have shown that several cytokines and proteins, such as $\mathrm{E}$-cadherin, $\mathrm{N}$-cadherin, vimentin, and fibronectin, are involved in the EMT process of tumor cells. Some lncRNAs can also significantly affect the EMT process of tumor cells. For example, lncRNA LINP1 can significantly inhibit TGF- $\beta$-induced EMT of lung cancer, thereby controlling the migration and invasion of cancer cells (25). LINC00673 can also regulate the proliferation, migration, invasion, and EMT of lung cancer through sponging miR-150-5p (26). The results of this study showed that knockdown of LINC00115 could significantly inhibit the invasion and migration of lung cancer cells. Further molecular mechanism studies showed that knockdown of LINC00115 significantly inhibited the expression of the EMT-related proteins N-cadherin, vimentin, and fibronectin, and upregulated the expression of E-cadherin. In the tumor microenvironment, fibronectin activates the TLR4-dependent inflammatory response in lung cancer cells, thereby leading to the upregulation of fibroinflammatory cytokines by activating the TLR4/ 


\section{Page 10 of 11}

NF- $\kappa \mathrm{B}$ signaling pathway (27). Therefore, LINC00115 may be involved in the regulation of the lung cancer microenvironment and the inflammatory response of tumors. However, it is still not clear how LINC00115 regulates the expression of downstream genes. Based on previous studies, we predicted that LINC00115 may act as a ceRNA to sequester miRNA, leading to the liberation of corresponding miRNA-targeted transcripts. So, we will further explore the molecular mechanisms of LINC00115 in the regulation of downstream genes.

In conclusion, this study found that lung cancer patients with high expression of LINC00115 had a shorter overall survival, and knockdown of LINC00115 could significantly inhibit the proliferation, clone formation, invasion, and migration of lung cancer cells. Knockdown of LINC00115 also significantly inhibited the expression of EMT-related genes. The results of this study reveal the molecular mechanisms of LINC00115 in the occurrence and development of lung cancer, and provide a molecular target and theoretical basis for the early diagnosis and targeted therapy of lung cancer.

\section{Acknowledgments}

Funding: Suzhou Science Technology Development Plan Project (SYS2-01730) and the National Natural Science Foundation of China (grant No. 81702806).

\section{Footnote}

Reporting Checklist: The authors have completed the ARRIVE reporting checklist. Available at https://dx.doi. org/10.21037/atm-21-3724

Data Sharing Statement: Available at https://dx.doi. org/10.21037/atm-21-3724

Conflicts of Interest: All authors have completed the ICMJE uniform disclosure form (available at https://dx.doi. org/10.21037/atm-21-3724). The authors have no conflicts of interest to declare.

Ethical Statement: The authors are accountable for all aspects of the work in ensuring that questions related to the accuracy or integrity of any part of the work are appropriately investigated and resolved. All procedures performed in this study involving human participants were in accordance with the Declaration of Helsinki

\section{Shao et al. LINC00115 inhibits the proliferation of lung cancer cells}

(as revised in 2013). The study was approved by Tumor Hospital Affiliated to Nantong University (No. 20516215) and informed consent was taken from all the patients. Experiments were performed under a project license (No.: 51815674) granted by Tumor Hospital Affiliated to Nantong University, in compliance with Chinese guidelines for the care and use of animals.

Open Access Statement: This is an Open Access article distributed in accordance with the Creative Commons Attribution-NonCommercial-NoDerivs 4.0 International License (CC BY-NC-ND 4.0), which permits the noncommercial replication and distribution of the article with the strict proviso that no changes or edits are made and the original work is properly cited (including links to both the formal publication through the relevant DOI and the license). See: https://creativecommons.org/licenses/by-nc-nd/4.0/.

\section{References}

1. Schabath MB, Cote ML. Cancer progress and priorities: lung cancer. Cancer Epidemiol Biomarkers Prev 2019;28:1563-79.

2. Rachow T, Schiffl H, Lang SM. Risk of lung cancer and renin-angiotensin blockade: a concise review. J Cancer Res Clin Oncol 2021;147:195-204.

3. Cao M, Chen W. Epidemiology of lung cancer in China. Thorac Cancer 2019;10:3-7.

4. Xing DF, Xu CD, Liao XY, et al. Spatial association between outdoor air pollution and lung cancer incidence in China. BMC Public Health 2019;19:1377.

5. Dubin S, Griffin D. Lung cancer in non-smokers. Mo Med 2020;117:375-9.

6. Wang Y, Zou S, Zhao Z, et al. New insights into smallcell lung cancer development and therapy. Cell Biol Int 2020;44:1564-76.

7. Maynard A, McCoach CE, Rotow JK, et al. Therapyinduced evolution of human lung cancer revealed by single-cell RNA sequencing. Cell 2020;182:1232-51.e22.

8. Jathar S, Kumar V, Srivastava J, et al. Technological Developments in lncRNA Biology. Adv Exp Med Biol 2017;1008:283-323.

9. Hu J, Wang T, Chen Q. Competitive endogenous RNA network identifies four long non-coding RNA signature as a candidate prognostic biomarker for lung adenocarcinoma. Transl Cancer Res 2019;8:1046-64.

10. Zhao Z, Sun W, Guo Z, et al. Mechanisms of lncRNA/ microRNA interactions in angiogenesis. Life Sci 
2020;254:116900

11. Liao K, Xu J, Yang W, et al. The research progress of LncRNA involved in the regulation of inflammatory diseases. Mol Immunol 2018;101:182-8.

12. Wang H, Hu A, Liang $Y$, et al. Genome-wide analysis of long non-coding RNA expression profile in lung adenocarcinoma compared to spinal metastasis. Ann Transl Med 2020;8:1516.

13. Fan CN, Ma L, Liu N. Systematic analysis of lncRNAmiRNA-mRNA competing endogenous RNA network identifies four-lncRNA signature as a prognostic biomarker for breast cancer. J Transl Med 2018;16:264.

14. Pan J, Fang S, Tian H, et al. lncRNA JPX/miR-33a-5p/ Twist1 axis regulates tumorigenesis and metastasis of lung cancer by activating $\mathrm{Wnt} / \beta$-catenin signaling. Mol Cancer 2020;19:9.

15. Zhao Y, Feng C, Li Y, et al. LncRNA H19 promotes lung cancer proliferation and metastasis by inhibiting miR-200a function. Mol Cell Biochem 2019;460:1-8.

16. Wu Y, Li L, Wang Q, et al. LINC00511 promotes lung squamous cell carcinoma proliferation and migration via inhibiting miR-150-5p and activating TADA1. Transl Lung Cancer Res 2020;9:1138-48.

17. Liu Z, Ning Z, Lu H, et al. Long non-coding RNA RFPL3S is a novel prognostic biomarker in lung cancer. Oncol Lett 2020;20:1270-80.

18. Feng W, Li B, Wang J, et al. Long Non-coding RNA LINC00115 contributes to the progression of colorectal cancer by targeting miR-489-3p via the PI3K/AKT/ mTOR pathway. Front Genet 2020;11:567630.

19. Yuan C, Luo X, Duan S, et al. Long noncoding RNA

Cite this article as: Shao L, Yu Q, Lu X, Zhang X, Zhuang Z. Downregulation of LINC00115 inhibits the proliferation and invasion of lung cancer cells in vivo and in vivo. Ann Transl Med 2021;9(15):1256. doi: 10.21037/atm-21-3724
LINC00115 promotes breast cancer metastasis by inhibiting miR-7. FEBS Open Bio 2020;10:1230-7.

20. Lai XN, Li J, Tang LB, et al. MiRNAs and lncRNAs: dual roles in TGF- $\beta$ signaling-regulated metastasis in lung cancer. Int J Mol Sci 2020;21:1193.

21. Fan T, Sun N, He J. Exosome-derived lncRNAs in lung cancer. Front Oncol 2020;10:1728.

22. Ginn L, Shi L, Montagna M, et al. LncRNAs in nonsmall-cell lung cancer. Noncoding RNA 2020;6:25.

23. Hua Q, Mi B, Xu F, et al. Hypoxia-induced lncRNAAC020978 promotes proliferation and glycolytic metabolism of non-small cell lung cancer by regulating PKM2/HIF-1 $\alpha$ axis. Theranostics 2020;10:4762-78.

24. Park SM, Choi EY, Bae DH, et al. The lncRNA EPEL promotes lung cancer cell proliferation through E2F target activation. Cell Physiol Biochem 2018;45:1270-83.

25. Zhang C, Hao Y, Wang Y, et al. TGF- $\beta /$ SMAD4regulated lncRNA-LINP1 inhibits epithelial-mesenchymal transition in lung cancer. Int J Biol Sci 2018;14:1715-23.

26. Lu W, Zhang H, Niu Y, et al. Long non-coding RNA linc00673 regulated non-small cell lung cancer proliferation, migration, invasion and epithelial mesenchymal transition by sponging miR-150-5p. Mol Cancer 2017;16:118.

27. Cho C, Horzempa C, Longo CM, et al. Fibronectin in the tumor microenvironment activates a TLR4-dependent inflammatory response in lung cancer cells. J Cancer 2020;11:3099-105.

(English Language Editor: C. Betlzar) 